\title{
Engagement anerkennen
}

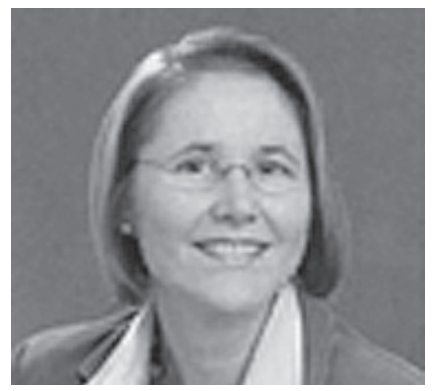

VON GABRIELE LANG

Gabriele Lang ist Diplom-Sozialarbeiterin, Systemischer Coach SG und staatlich geprüfte Hauswirtschaftliche Betriebsleiterin. Mit der Unternehmensberatung "Fokus Ehrenamt " gestaltet und begleitet sie Lernprozesse in Organisationen, damit Organisationen selbst Ehrenamtliche erfolgreich gewinnen und begleiten können. www.fokus-ehrenamt.de

\author{
Organisationen, die mit Ehrenamtlichen arbeiten, \\ brauchen auf Dauer eine Anerkennungskultur \\ für freiwilliges soziales Engagement. Um dies im \\ Betriebsalltag praktisch umzusetzen, braucht es ein \\ organisationsspezifisches Konzept, um angemessene \\ Formen der Würdigung jenseits der Bezahlung zu finden.
}

Der Begriff »Anerkennungskultur« bezieht sich auf das Miteinander in einer Organisation oder einem Projekt, auf die Art und Weise, wie Hauptamtliche, Führungskräfte und Mitarbeitende sowie Ehrenamtliche (1), sich gegenseitig Anerkennung und Wertschätzung, für ihre Haltung und Leistung zuteilwerden lassen.

Der Freiwilligensurvey der Bundesregierung hat festgestellt, dass das »vergütete « freiwillige Engagement in den vergangenen Jahren zugenommen hat. Das bedeutet, dass immer mehr Handlungsräume für Freiwillige geschaffen werden, die eine »Vergütung « als Anerkennung vorsehen. Diese Vergütung wird von den Freiwilligen nicht als »Lohn« (2) im engeren Sinne eingeordnet, sondern als Teil der Anerkennung gesehen, dem sie eine höhere Bedeutung ten, als Ausgleich für ihr Engagement erlebt? Welche Haltungen brauchen Führungskräfte, Mitarbeitende und Ehrenamtliche, damit sich eine Kultur der Anerkennung in einer Organisation entwickeln kann? Wenn Anerkennung gelingt, sind Ehrenamtliche, auch ohne Vergütung, zufrieden, engagieren sich verbindlich und bleiben der Organisation lange erhalten.

Das Thema Anerkennung spielt in vielen Bereichen unseres Zusammenlebens eine elementare Rolle. Dabei geht es um die Beziehungsebene zwischen den Menschen. Deshalb handelt es sich um ein grundlegendes Thema der systemischen Beratung. Die systemischen Aspekte des Beitrags gehen auf die Entwicklungsarbeiten von Matthias Varga von Kibèd und Insa Sparrer am SySt ${ }^{\circledR}$ Institut in München zurück.

\section{"Die innere Haltung von Fach- und Führungskräften zum Ehrenamt bleibt Ehrenamtlichen nicht verborgen«}

zumessen als anderen gängigen Anerkennungsformen, wie zum Beispiel öffentlichen Würdigungen.

Wie aber gelingt eine Kultur der Anerkennung bei einem nicht vergüteten Ehrenamt? Was genau wird von Ehrenamtlichen, die keine Vergütung erhal-

\section{Erste These: Die Beziehung zwischen der Organisation und Ehrenamtlichen braucht einen Ausgleich}

Was genau geschieht auf der Beziehungsebene, wenn sich Bürgerinnen und Bür- 


\begin{tabular}{|c|c|c|c|}
\hline Tätigkeitsform ${ }^{32}$ & Vergütungsform & $\begin{array}{c}\text { Beitragsfreiheit } \\
\text { (gesetzliche KV, } \\
\text { PV, RV, AV) }\end{array}$ & $\begin{array}{l}\text { Steuerfreiheit/- } \\
\text { begünstigung }\end{array}$ \\
\hline \multicolumn{4}{|l|}{$\begin{array}{l}\text { Hauptberufliche Tätigkeit } \\
\text { mit Gemeinwohlbezug }\end{array}$} \\
\hline - $\quad$ als Arbeitnehmer & Arbeitslohn, - entgelt & nein & nein \\
\hline - als Selbstständiger & Honorar & $\begin{array}{l}\text { ja (aber Ausnahmen } \\
\text { in der RV, § 2 SGB VI) }\end{array}$ & nein \\
\hline \multicolumn{4}{|l|}{$\begin{array}{l}\text { Nebenberufliche Tätigkeit } \\
\text { mit Gemeinwohlbezug }\end{array}$} \\
\hline - $\quad$ § $3 \mathrm{Nr} .26 \mathrm{EStG}$ & $\begin{array}{l}\text { Obbungsleiterpauscha- } \\
\text { le, Honorar }\end{array}$ & ja & \begin{tabular}{|l|} 
Ja, bismax \\
2100€/Jahr
\end{tabular} \\
\hline - $\S 3 \mathrm{Nr} .26 \mathrm{a}$ EStG & Ehrenamtspauschale & ja & $\begin{array}{l}\text { Ja, bis max. } 500 \\
\in / \text { Jahr } \\
\end{array}$ \\
\hline - $\S 3 \mathrm{Nr} .26 \mathrm{~b}$ EStG & Betreuervergütung & ja & \begin{tabular}{|l|} 
Ja, bismax \\
$2100 \in / J$ ahr \\
\end{tabular} \\
\hline $\begin{array}{ll}\text { - } & \text { Geringfügige } \\
& \text { Beschäftigung }\end{array}$ & Arbeitslohn,-entgelt & $\mathrm{ja}^{33}$ & nein $^{34}$ \\
\hline \multicolumn{4}{|l|}{$\begin{array}{l}\text { Qualifizierende gemein- } \\
\text { wohlbezogene Tätigkeiten }\end{array}$} \\
\hline $\begin{array}{l}\text { Bundesfreiwilligendienst, } \\
\text { FSJ }\end{array}$ & $\begin{array}{l}\text { Taschengeld, } \\
\text { ggf. Unterkunft oder } \\
\text { Unterkunftszuschuss, } \\
\text { ggf. Verpflegung oder } \\
\text { Verpflegungsgeld }\end{array}$ & nein & nein \\
\hline \multicolumn{4}{|l|}{ Freiwilliges Engagement } \\
\hline & $\begin{array}{l}\text { Auslagenersatz gem. } \\
\$ 670 \text { BGB, pauschaler } \\
\text { Auslagenersatz }\end{array}$ & ja & ja \\
\hline
\end{tabular}

32 Tätigkeitsformen entsprechend der Einteilung von Klie, T/Stemmer, P.Negner, M.: Untersuchung zur Monetarisierung von Ehrenamt und Bürgerschaftlichem Engagement in Baden-Württemberg im Auftrag des Ministeriums für Arbeit und Soziales Baden-Württemberg, Stuttgart 2010

33 aber pauschale Beitragsleistung des Arbeitgebers

34 Zahlung eines Pauschsteuersatzes möglich

Der Paritätische Wohlfahrtsverband hat eine Arbeitshilfe zum Einsatz von Ehrenamtlichen vorgelegt. Die Broschüre beleuchtet arbeits-, sozialversicherungs- und steuerrechtlicher Aspekte der Mitwirkung von freiwilligen Mitarbeiterinnen und Mitarbeitern bei gemeinnützigen Trägern in der Sozialwirtschaft. Ein Schwerpunkt der Darstellung liegt in der Abgrenzung der ehrenamtlichen Tätigkeit gegenüber sozialversicherungsund lohnsteuerpflichtigen Beschäftigungsverhältnissen. Im Anhang der 50-seitigen Broschüre finden sich u. a. Mustervereinbarungen zum Einsatz von Ehrenamtlichen und Übungsleitern. Die Arbeitshilfe kann als PDF-Dokument kostenlos von der Website des Paritätischen heruntergeladen werden.

www.der-paritaetische.de/uploads/tx_pdforder/broschuere_ehrenamt_web.pdf

ger ehrenamtlich engagieren? In dem sie ihre Zeit, Zuwendung, Fähigkeiten, ihr Wissen und vieles mehr freiwillig, also ohne vertragliche Verpflichtung einbringen, werden sie zu Leistungserbringern und die Organisation und die Kunden der Organisation zur Leistungsempfängerin. Der Leistung steht keine Gegenleistung, beispielsweise ein Honorar, als Ausgleich gegenüber. Ohne Ausgleich gerät die Beziehung zwischen der Organisation und den Ehrenamtlichen in eine Unausgeglichenheit, die für beide Seiten kein befriedigender Zustand darstellt.

\section{Zweite These: Die Organisation muss die Pflicht zum Ausgleich anerkennen}

"Ehrenamtlichen etwas schuldig zu sein « - so empfinden es viele Führungskräfte und Mitarbeitende, die mit Ehrenamtlichen zu tun haben. Ein solcher Satz formuliert indirekt den Wunsch nach Ausgleich, um die Leistung von Ehrenamtlichen annehmen zu können.

Der Begriff der »Schuld « ist im Deutschen mehrdeutig. Wenn Hauptamtliche sich Ehrenamtlichen gegenüber »schul- dig « fühlen, geht es nicht um eine ethische Schuld (»gut " oder »böse«), sondern um eine ökonomische (Schuld und Ausgleich) Betrachtungsweise von Schuld. (3) Schulden bei einer Bank sind nicht verwerflich oder moralisch anrüchig, aber es braucht eine Verpflichtung vonseiten des Darlehensnehmers, dass er der Bank das Geld zurückbezahlt und die Schulden ausgleicht. Durch diese Ausgleichsverpflichtung wird die Geschäftsbeziehung zwischen Bank und Darlehensnehmer gleichgestellt.

In ähnlicher Weise braucht es eine Verpflichtung vonseiten der Führungskräfte und Mitarbeitenden gegenüber Ehrenamtlichen, für Ausgleich in der Beziehung zu sorgen, es braucht eine Anerkennung der Ausgleichsverpflichtung. Diese zunächst innere Haltung bleibt den Ehrenamtlichen nicht verborgen, beeinflusst das aktive Handeln ihnen gegenüber in Wort und Tat, schafft Rahmenbedingungen, in denen sich Ehrenamtliche und ihr Engagement optimal entfalten und versetzt die Beziehung zueinander wieder in einen "geraden Stand « (4).

Woran könnte für Ehrenamtliche in der Alltagspraxis deutlich werden, dass Führungskräfte und Mitarbeitende ihre Ausgleichsverpflichtung ihnen gegenüber anerkennen? Es könnte deutlich werden, zum Beispiel, wenn Ehrenamtliche

- Zuwendung und Freundlichkeit und eine wertschätzende Wahrnehmung von allen Mitarbeiterinnen und Mitarbeitern im Haus entgegengebracht wird

- eine Erstattung, der im Engagement entstehenden Kosten, beispielsweise Fahrtkosten, angeboten wird

- einen kostenlosen Zugang zu Wissen (im Rahmen des Engagements) und verlässlichen Zugang zu hausinternen Informationen gewährleistet wird

- an wichtigen Entscheidungen im Haus mitwirken, ihre Sichtweisen ernstgenommen und systematisch ihre Erfahrungen eingeholt werden

- Gelegenheit zum Erfahrungsaustausch mit anderen Ehrenamtlichen im Haus ermöglicht wird

- Freiräume erhalten, in denen sie ihre Neigungen entfalten können

- eine Förderung ihrer Fähigkeiten und Talente erfahren, in dem ihnen beispielsweise ein Zugang zu ehrenamt- 


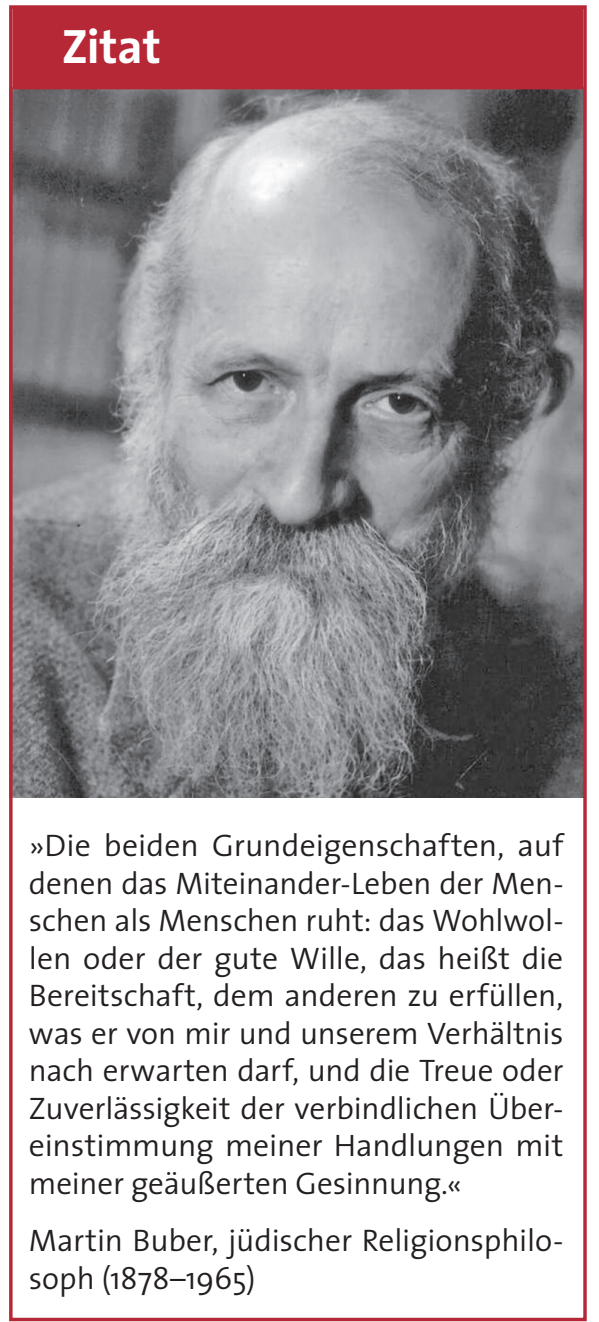

lichen Führungspositionen eröffnet wird

- Schutz und Geborgenheit erhalten, die die Organisation oder Gruppe geben kann

\section{Dritte These: Die \\ Ausgleichsleistung macht den Ausgleich perfekt}

Wenn die Anerkennung der Ausgleichsverpflichtung den eigentlichen Ausgleich bewirkt und die Beziehungsbalance wieder herstellt, könnte man auf die Idee kommen, dass damit dem Ausgleich genüge getan ist. So ist es nicht. Auch zwischen dem Darlehensnehmer und der Bank genügt nicht nur der unterschriebene Darlehensvertrag, sondern erst wenn die Ratenzahlungen bei der Bank eingehen, wird der Ausgleich wirksam.

So wie der Siegellack einer Urkunde Gültigkeit verleiht, vermittelt die Ausgleichsleistung dem Ehrenamtlichen die Ernsthaftigkeit der Anerkennung. Eine angemessene und passende Aus- gleichsleistung muss in der Form und im Umfang zum Engagement und zum Empfänger passen. (5)

Wie aber lässt sich in der Praxis eine passende Ausgleichsleistung für Ehrenamtliche finden? In jedem Fall geht es darum, sich in Ehrenamtliche hineinzuversetzen. Dafür brauchen Verantwortliche und Mitarbeitende Wahrnehmungsfähigkeit und Mitgefühl, um das passende "Dankeschön« zu finden. Zum Beispiel könnten einer Ehrenamtlichen, die das Rampenlicht einer öffentlichen Ehrung durch den Landrat scheut, Blumen und Urkunde in einem persönlicheren Rahmen überreicht werden.

\section{Vierte These: Auch Ehrenamtliche haben eine Ausgleichsverpflichtung}

Die Fachdiskussion »Anerkennungskultur für Ehrenamtliche « bezieht sich in der Regel nur auf die Ausgleichsverpflichtung der Organisation gegenüber den Ehrenamtlichen. Dass die Organisation als Leistungserbringerin gegenüber den Ehrenamtlichen auftritt, gerät manchmal aus dem Blickfeld.

Ein ehrenamtliches Engagement eröffnet Bürgerinnen und Bürgern die Möglichkeit, eine sinnstiftende Tätigkeit auszuüben, die eigenen Fähigkeiten einzusetzen und weiterzuentwickeln und neue soziale Kontakte zu knüpfen. Dadurch werden Ehrenamtliche zu Leistungsempfängern. Bei der Anerkennung der Ausgleichsverpflichtung handelt es sich somit um eine wechselseitige Verpflichtung für Mitarbeitende sowie Ehrenamtliche.

Woran könnten Führungskräfte und Mitarbeiter merken, dass Ehrenamtliche es mit der Anerkennung ihrer Ausgleichsverpflichtung ernst meinen? Beispielsweise wenn

- die Organisation als Leistungserbringerin wahrgenommen und anerkannt wird

- Ehrenamtliche sich verbindlich und verlässlich engagieren

- Ehrenamtliche sich um eine gute $\mathrm{Zu}$ sammenarbeit mit Hauptamtlichen bemühen

- Ehrenamtliche die Strukturen der Organisation akzeptieren und sich in die Kultur des Hauses einfügen

- Ehrenamtliche bereit sind, an Weiterbildung teilzunehmen.

\section{Anmerkungen}

(1) Der Begriff »Ehrenamtlicher « ist von der Autorin im Artikel bewusst gewählt, weil er heute noch am ehesten vermuten lässt, dass es sich um ein unvergütetes Engagement handelt. Durch die Einführung der Freiwilligendienste lässt sich der Begriff »Freiwilliger " kaum noch als Überbegriff für ein vergütetes und nichtvergütetes Engagement verwenden. (2) Thomas Klie, Philipp Stemmer, Martina Wegner, Untersuchung zur Monetarisierung von Ehrenamt und Bürgerschaftlichem Engagement in BadenWürttemberg, November 2009.

(3) Insa Sparrer, Matthias Varga von Kibéd, Klare Sicht im Blindflug, 2010, S. 68.

(4) Matthias Varga von Kibéd, private Seminarmitschrift, November 2009.

(5) Matthias Varga von Kibéd, private Seminarmitschrift, November 2009.

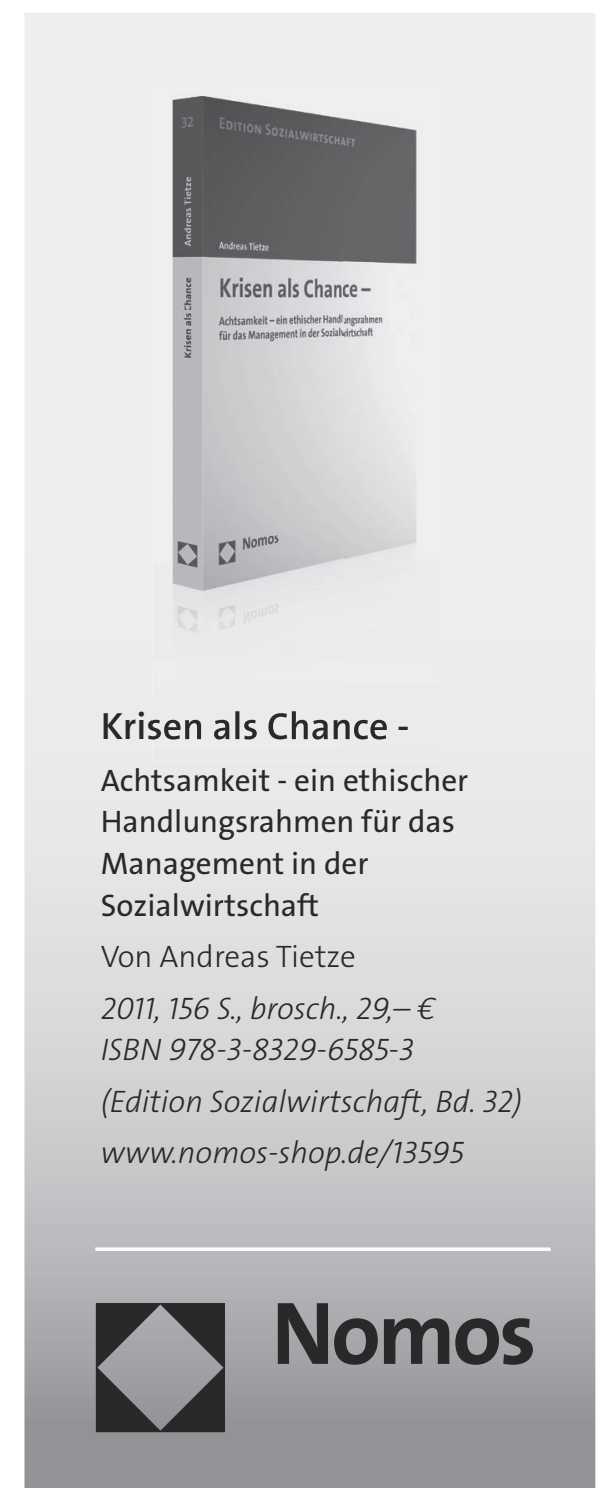

